\title{
Association between Gene Polymorphism of Manganese Superoxide Dismutase and Prostate Cancer Risk
}

\author{
Ayşe Eken, ${ }^{1,2}$ Onur Erdem, ${ }^{2}$ Zorica Arsova-Sarafinovska, ${ }^{3}$ Cemal Akay, ${ }^{2}$ Ahmet Sayal, ${ }^{2}$ \\ Nadica Matevska, ${ }^{4}$ Ljubica Suturkova, ${ }^{4}$ Koray Erten, ${ }^{5}$ Yaşar Özö̈k, ${ }^{5}$ \\ Aleksandar Dimovski, ${ }^{4}$ and Ahmet Aydin ${ }^{2,6}$ \\ ${ }^{1}$ Department of Toxicology, Erciyes University, Faculty of Pharmacy, Kayseri, Turkey \\ ${ }^{2}$ Department of Toxicology, Gulhane Military Medical Academy, Ankara, Turkey; E-mail: ahmetaydin30@hotmail.com \\ ${ }^{3}$ Department for Quality Control of Medicine, Institute of Public Health of the Republic of Macedonia, Skopje, Republic of Macedonia \\ ${ }^{4}$ Faculty of Pharmacy, University of St. Cyril and Methodius, Skopje, Republic of Macedonia \\ ${ }^{5}$ Department of Urology, Gulhane Military Medical Academy, Ankara, Turkey \\ ${ }^{6}$ Department of Toxicology, Faculty of Pharmacy, Yeditepe University, Istanbul, Turkey
}

Received 26 September 2012; revised 21 November 2012; accepted 8 December 2012

\begin{abstract}
Manganese superoxide dismutase (MnSOD) is the most effective antioxidant enzyme in $\mathrm{mi}-$ tochondria and protects cells from reactive oxygen species-induced oxidative damage. The aim of this study was to investigate the association between $\mathrm{Mn}$ $S O D$ Ala-9Val gene polymorphism and prostate cancer (PCa) risk in Turkish men with prostate cancer. 33 patients with PCa and 81 control individuals were included in the study. We observed an association between MnSOD Ala/Ala frequency and a higher PCa risk. In addition, we found that the increased risk of early-onset PCa (under age of 65) in the men homozygous for Ala allele was higher than the men homozygous for Val allele. However, we determined that MnSOD Ala-9Val genotype was not associated with the aggressiveness of the disease. The results of our study suggest that $M n S O D$ Ala/Ala genotype may influence on early-onset of PCa patients, but no effect on subsequent development of the disease in Turkish men. However, our study has a limitation that is small numbers of individuals for cases and controls. Therefore, the presented study limited our statistical power to fully investigate the gene polymorphism on cancer risk. () 2013 Wiley Periodicals, Inc. J Biochem
\end{abstract}

Correspondence to: Ahmet Aydin.

Contract Grant Sponsor: Turkish Scientific and Technical Research Association (TUBITAK) (to AA).

Contract Grant Sponsor: Ministry of Education and Science of the Republic of Macedonia (AJD and AS).

(c) 2013 Wiley Periodicals, Inc.
Mol Toxicol 27:213-218, 2013; View this article online at wileyonlinelibrary.com. DOI 10.1002/jbt.21472

KEYWORDS: Genetic Polymorphism; Manganese Superoxide Dismutase; Prostatic Cancer; Antioxidant Effect; Reactive Oxygen Species

\section{INTRODUCTION}

Manganese superoxide dismutase (MnSOD) is the most effective antioxidant enzyme in mitochondria [1] and has an important role in protecting cells from reactive oxygen species (ROS)-induced oxidative damage $[2,3]$. It has been suggested that MnSOD (Ala-9Val) gene polymorphism may alter the secondary structure of the protein [4-6] and affects transport of MnSOD enzyme into the mitochondria $[7,8]$.

It has been indicated that the Ala-containing MnSOD is transported more efficiently through the mitochondrial membrane, suggesting that the individuals with the homozygous AA genotype may have higher MnSOD activity in comparison with those with the $\mathrm{Val} / \mathrm{Val}$ and $\mathrm{Val} / \mathrm{Ala}$ genotype $[9,10]$. The MnSOD Ala variant showed normal transport and generated 30$40 \%$ more active protein than did the Val variant in mitochondria [7]. Since the superoxide anion is dismutated by MnSOD, a potential source of oxidative damage, one can predict that the MnSOD Val allele (but not the Ala allele) may lead to an increased risk of cancer. However, if some individuals have decreased capacity to remove hydrogen peroxide by enzymes of catalase 